\title{
An experimental comparison of three- versus four-surface phenomenal transparency
}

\author{
SERGIO CESARE MASIN \\ University of Padua, Padua, Italy
}

\begin{abstract}
The three-surface transparency occurs when an object seen through a transparency does not jut out under the transparent surface. The four-surface transparency occurs when the object juts out. Observers rated the density of the transparent surface in both kinds of transparency. The results seem to show that the topological diversity between the two kinds of transparency has no functional significance. The stimulus conditions ruling the generation of judgments were detected and expressed in terms of an algebraic model.
\end{abstract}

There are two kinds of basic proximal stimulus patterns that produce phenomenal transparency. Figure 1 depicts these two possibilities when an object, the small square, is looked at through a filter, the big square. Figure 1a represents the case in which the object does not jut out under the filter; Figure 1b represents the case in which an object juts out under the filter. In Figure 1a, there are three proximal surfaces, yielding the kind of transparency called here the "three-surface" phenomenal transparency. In Figure 1b, there are four proximal surfaces, yielding the "four-surface" phenomenal transparency.

Theoretical interest has so far been addressed only to the four-surface transparency with achromatic colors. Metelli $(1970,1974 a, 1974 b, 1975$; see also Masin, 1978) developed the following model. Phenomenal transparency entails the generation of overlapping surfaces. These surfaces are the transparent layer and the surfaces (the background and the objects on the background) seen through it. MooreHeider (1933) hypothesized that, when transparency is perceived, the reduction colors ${ }^{1}$ split, so to speak, into the colors of the corresponding overlapping surfaces. Let $a, p, q$, and $b$ be the achromatic reduction colors corresponding to the proximal surfaces $\mathbf{A}, \mathbf{P}$, $Q$, and B in Figure 1b. On the basis of Moore-Heider's hypothesis, Metelli (1982) assumed ${ }^{2}$ that $p$ split into $a$ and $t$, where $t$ is the color of the transparent layer, and $q$ split into $b$ and $t$, following $a$ rule that is the opposite of Talbot's law. The model is stated as follows

$$
\begin{aligned}
& \mathrm{p}=\alpha_{1} \mathrm{a}+\left(1-\alpha_{1}\right) \mathrm{t}_{1}, \\
& \mathrm{q}=\alpha_{2} \mathrm{~b}+\left(1-\alpha_{2}\right) \mathrm{t}_{2},
\end{aligned}
$$

I wish to thank Jacob Beck and Fabio Metelli for useful comments. Correspondence may be addressed to me at Istituto di Psicologia, Piazza Capitaniato 3, 35139 Padova, Italy.
A

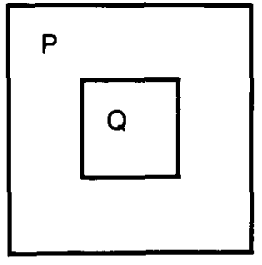

a

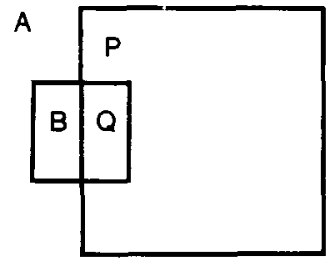

b

Figure 1. Illustration of the three- (a) and four-surface (b) transparency. The three-surface transparency occurs when the object (the small square) does not jut out under the transparent surface (the big square). The four-surface transparency occurs when the object juts out.

where the weights $\alpha_{1}$ and $\alpha_{2}$ are interpreted as coefficients expressing how transparent the transparent layer is. The weight $\alpha_{1}$ (the symbol $t_{1}$ ) refers to the degree of perceived transparency (to the color) over the background, and $a_{2}\left(t_{2}\right)$ refers to the degree of transparency (to the color) over the part of the object under the transparent surface. ${ }^{3}$

We must ask now whether Model 1 applies also to the three-surface transparency (Figure 1a). A close following of Model 1 leads to the assertion that $p$ splits into $a$ and $t$, and $q$ splits into $x$ and $t$, where $x$ is the color of the object seen through the transparency. Since $x$ is an unknown, Model 1 is inapplicable, on principle, to the three-surface transparency. Moreover, Masin (in press) showed experimentally that $\mathrm{x}=\mathrm{q}$, which means that the process of the splitting of reduction colors (Moore-Heider, 1933) does not occur in the three-surface transparency.

It is possible, however, that the splitting of colors does occur in the four-surface transparency. It is clear that if this splitting occurs, it must be ascribed to the presence of the part of the object jutting out under the transparent surface (B in Figure 1b). It is, therefore, of theoretical importance to check whether this part has a functional influence on the degree of perceived transparency in the transparent layer. 


\section{EXPERIMENT 1}

\section{Method}

Observers. The observers were 33 students and members of the Institute of Psychology.

Stimuli. Each of five (Kodak Wratten No. 96) achromatic gelatine filters, $72 \times 76 \mathrm{~mm}$, with a transmittance of $.1, .2, .3, .5$, or .8 , was placed in between two exactly superimposed $1.5 \times 220$ $\times 275 \mathrm{~mm}$ glass sheets. The reflectance of the filters was about .05 . The two glass sheets, with the filter between them, were put in a wooden rectangular frame. A small, $23 \times 23 \mathrm{~mm}$ acrhomatic square, with a reflectance $.04, .11, .19, .26, .31, .40, .52, .67$, or .87 (NCS papers, Sweden), was stuck at the center of each of nine $200 \times 200 \mathrm{~mm}$ achromatic backgrounds, all with the same .31 , reflectance.

The observer sat at a small table provided with a chinrest to keep the eyes level with the stimulus. On another table, the background with the small square on it was placed at $3.1 \mathrm{~m}$ from the observer's eyes. The wooden frames, with the filters suspended in the middle of them, were placed at $2.9 \mathrm{~m}$, between the small square on the background and the observer's eyes. The observer viewed the scene binocularly. The background could be placed in two positions. In one position, the small square on the background was perceived in the middle of the filter (three-surface transparency, Figure 1a). In the other position, half of the square jutted out, on the left, under the filter (four-surface transparency, Figure 1b).

The wooden frame, parallel to the background, was tilted about 6 deg backwards so that the glass sheets reflected a uniform black curtain just above the observer's head, thus assuring no reflected image in the glass sheets. The light came from neon tubes on the ceiling. The illumination level was of $20 \mathrm{~lx}$.

Procedure. The observers were shown each of the 45 combinations of filter and square on the background once, with the background in one position. Then the 45 combinations were again shown once with the background in the other position. All the 45 combinations were shown in a random order that was different for each observer. The experimenter was hidden by a screen. The patterns were shown by removing another screen. Two groups of 15 observers each were formed. One group was first shown the three-surface transparency, and then the four-surface transparency. For the other group, this order was reversed.

For each combination, the observer was asked to assign a number to the filter according to its density and to write that number down on a page of a notebook (one number per page). $\mathrm{He} / \mathrm{she}$ was told to match the number to his/her subjective impression of the amount of density in the filter (that is, the greater the density, the larger the number, and the less the density, the smaller the number), and could use any number that seemed appropriate. When observers asked, they were told they could use both integers and fractions of whatever magnitude they thought appropriate, and that they were not limited to using fixed upper or lower numbers.

Before being given the instructions, the observer was shown seven photographs of a filter on a background of four black and white squares. The transmittance of the original filter decreased from photo 1 to photo 7. The experimenter described the figures by pointing out that the density of the filter increased progressively from photo 1 to photo 7, and that the greater the density, the less the visibility of the background through the filter. All observers promptly agreed with this description. Then they were shown, in the experimental apparatus, two examples of a combination of filter and square, one with a dense filter and one with a less dense filter (neither being of the greatest or of the least density). A session lasted $50 \mathrm{~min}$.

\section{Results}

All observers spontaneously used a fixed range of numerical responses. For 29 observers, the lower-end number was 0 and the upper end number was 100 $(\mathrm{N}=12), 10(5), 25(2), 1200,1000,250,200,80,50$, $40,30,20$, or $15(\mathrm{~N}=1$ for each of last 10 numbers). One observer used the range $30-40$. The data were linearly transformed so as to make the common range be $0-100$ for all observers. This processing is admissible because all observers, except one, used the same upper and lower end numbers for both kinds of transparency. For the exceptional subject, the first number lower than the larger number was used as an upper number.

A $2 \times 2 \times 5 \times 8$ analysis of variance was carried out using the normalized numerical responses as scores. The factors were, respectively, the group of observers, the kind of transparency, the transmittance, and the reflectance (the reflectance .31 was not included in the analysis, because it was a special case to be considered separately).

The analysis showed no significant difference between the two groups of observers $[F(1,28)=1.17]$. Figure $2 \mathrm{a}$ shows the results pooled over the two groups $(\mathbf{N}=30)$. The main effects due to the kind of transparency are not statistically different $[F(1,28)=1.98]$. The interaction between the kind of transparency and the reflectance is significant $[F(7,196)=9.59, p<.001]$.

This interaction shows in Figure $2 \mathrm{a}$ as a departure from the perfect overlapping of curves for the two kinds of transparency. This departure increases as the difference in lightness between the square and the background increases.

The group of 30 observers was formed by retaining in the group the observers who showed a pattern of results substantially similar to the one illustrated in Figure 2a. Three observers were excluded from the group because their results, represented in Figure 2b, showed no systematic pattern.

Figures $2 a$ and $2 b$ show that observers rated the filters as having an apparent density (results represented by squares) of less than the highest possible density (100) even when the square on the background had a reflectance (.31) equal to the reflectance of the background. In this case, none of the observers detected any figure or inhomogeneity on the background. They were consequently expected to perceive the filter as opaque (Fuchs, 1923) and to rate it as having the highest possible density, whatever its transmittance. However, only 19 of 30 observers (Figure $2 \mathrm{a}$ ) did perceive the filter as opaque. The remaining 11 observers plus the 3 observers excluded from the analysis (Figure $2 b$ ) perceived the filter as being transparent. A plausible reason for this is the following.

When a homogeneous filter is put on a homogeneous background, the filter appears to be opaque (Fuchs, 1923). Masin and Idone (1980) showed that, when the difference in lightness between the filter and the background is not too large, and when a favorable attitude is assumed, the filter may be perceived as transparent. The attitude towards perceiv- 

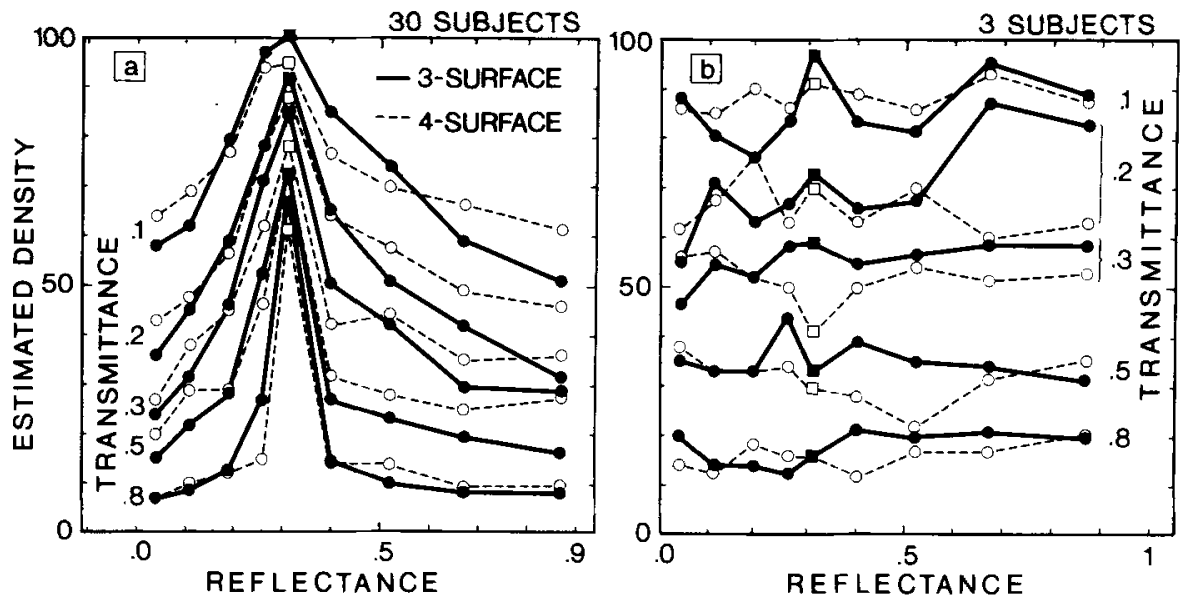

Figure 2. The results in Figure 2a show that apparent density of a transparent surface is a function of the difference in reflectance (and consequently in color) between the figure and the background. The reflectance of the figure is represented on the abscissa. The background had the reflectance .31 . Figure $2 b$ shows the results for three special observers, who rated the density both in the case of the three- (unbroken lines) and four-surface (dashed lines) transparency.

ing transparency was induced by means of instructions to the observer. It is reasonable to suppose that, in the present experiment, the attitude was induced objectively by the experimental procedure. Observers felt a certain uneasiness in rating the density of a filter when no figure was perceptible through it. The uneasiness was caused, they said, because the quality of the transparency was somehow different from that when a figure could be detected through the filter. The existence of this form of transparency, which might be called a "nonfunctional" transparency, has been independently reported by Tudor-Hart (1928, p. 283-284), Lauenstein (1943, p. 210), and Gibson (1975).

The results represented by squares in Figure 2 are therefore misleading in that they reflect responses both to opacity and to nonfunctional transparency. In order to check whether these kinds of responses were correlated with some other individual differences, the 19 observers who perceived opacity and the 11 observers who perceived nonfunctional transparency were assigned to two distinct groups, $A$ and B. An analysis of variance, in which the new factor was Group A vs. Group B, was then carried out.

The results are depicted in Figure 3. As may be seen, the curves for Group B $(\mathrm{N}=11)$ are much flatter than the curves for Group $A(N=19)$. The differences between the curves in the four graphs of Figure 3 are explained by significant interactions between kind of transparency and reflectance $[F(7,128)$ $=7.44, \mathrm{p}<.001]$, Group A vs. Group B and reflectance $[F(7,128)=18.82, p<.001]$, and Group $A$ vs. Group $B$, transmittance, and reflectance $[F(28,728)$ $=3.47, p<.001]$. The main effects due to the factor of Group A vs. Group B are not statistically different $[F(1,26)=1.64]$.

\section{Discussion}

Tudor-Hart (1928) seems to have been the first to show experimentally that apparent density of a filter, or an episcotister, increases as the sharpness of the contour of the object on the background decreases. ${ }^{4}$ Her finding is confirmed by the results depicted in Figure 2a.

The results in Figure $2 \mathrm{a}$ show that it makes a difference whether the case is one of a three- or a foursurface transparency. However, based on the assumption that apparent density depends upon the difference between $p$ and $q$ (Figure 1), the difference in results for the three- and four-surface transparencies can be explained, reasonably, in terms of simultaneous contrast. In fact, owing to simultaneous contrast produced by $B$ on $Q$, the difference $|p-q|$ in Figure $1 \mathrm{~b}$ increases (with respect to the difference $|\mathbf{p}-\mathbf{q}|$ in Figure 1a) as the difference $|\mathbf{b}-\mathbf{a}|$ increases. This could well explain the slight overestimation of apparent density in the case of the four-surface transparency for large differences between $a$ and $b$ and for low transmittances.

This conclusion is of theoretical importance, since it shows that the presence of region $B$ in Figure $1 \mathrm{~b}$ has no functional effect. Only the difference $|p-q|$ would be effective.

However, the absence of a pattern of results for three observers (Figure $2 b$ ) reveals the probable effect also of the difference, $|p-a|$, between the filter and the background. These results could be explained satisfactorily by assuming that the three observers 


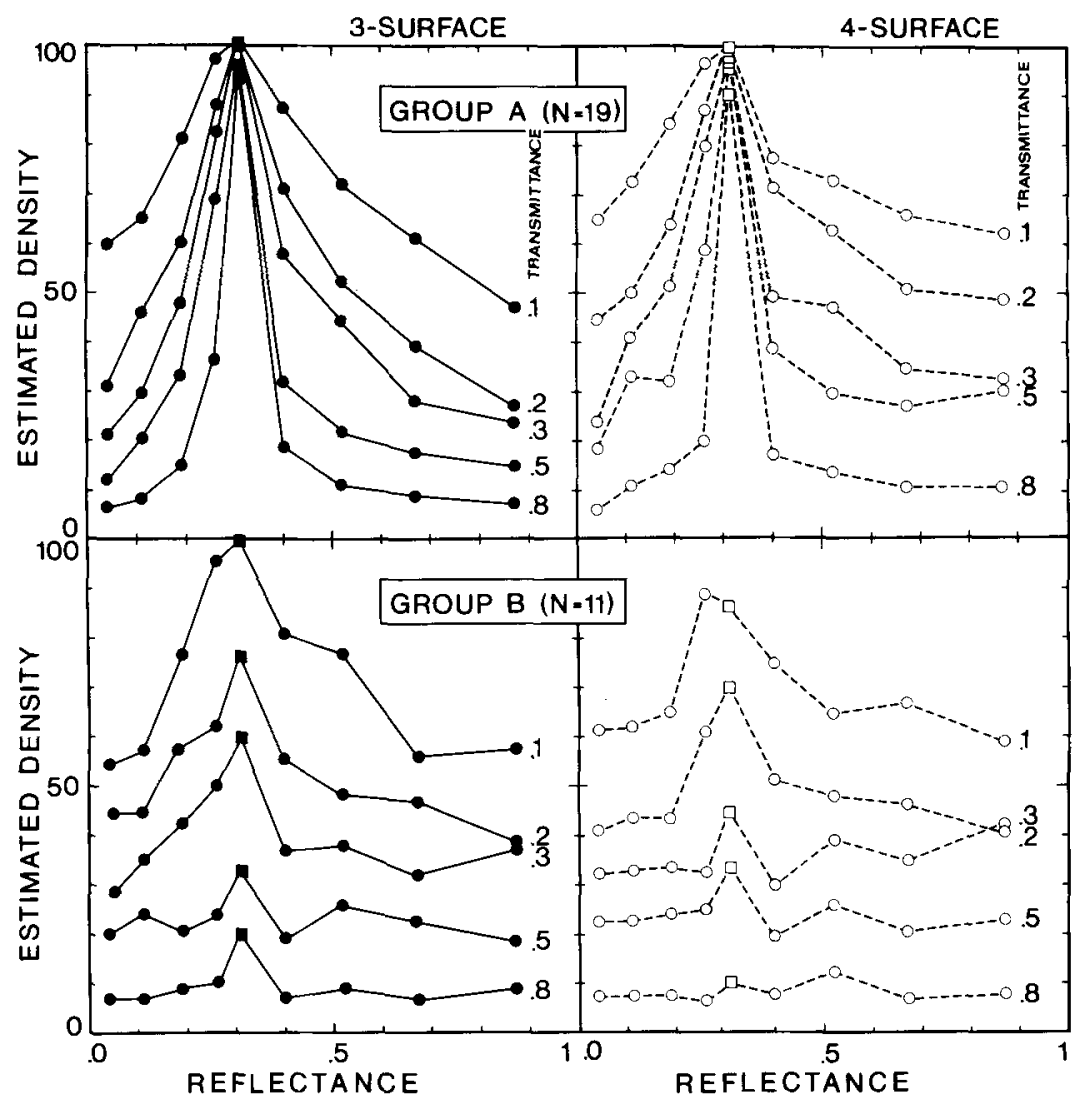

Figure 3. The same results as in Figure 2a when observers are subdivided in two subgroups according to whether or not they detected nonfunctional transparency.

rated the density only on the part of the filter over the background, while the other 30 observers rated the density of the filter over the figure. The three exceptional observers produced flat curves because, for a given filter, the difference $|\mathbf{p}-\mathbf{a}|$ was nearly the same for all reflectances of the figure.

If there are three extreme cases in which only the transparency of the filter on the background is rated, then, in a group of $\mathbf{3 0}$ observers, there must be extreme cases in which the transparency on only the figure is rated and also, reasonably, some mixed cases. A plausible criterion for selecting the extreme and mixed cases from the group of $\mathbf{3 0}$ observers is the following.

The occurrence of the impression of nonfunctional transparency depends only on the difference in color between the figure and the background (Masin \& Idone, 1980). In the group of 30 , the observers whose responses were based on this difference (i.e., $|\mathbf{p}-\mathbf{a}|$, when $p=q$ and $b=a)$ are likely to have rated nonfunctional transparency (mixed cases). It is also likely, although not necessary, that the observers who did not rate nonfunctional transparency were much more influenced by the difference $|p-q|$ than by the difference $|p-a|$ (extreme cases). Figure 3 represents the results for a subgroup $(N=19)$ that did not report nonfunctional transparency and for a subgroup $(\mathrm{N}=11)$ that did report nonfunctional transparency. The results from this analysis show that 19 of 33 observers seemed to be prevailingly influenced by the difference $|\mathbf{p}-\mathbf{q}|, 3$ observers were by the difference $|p-a|$, and 11 observers were by both differences.

\section{AN AVERAGING MODEL OF RATED COLOR DENSITY}

To sum up, the foregoing discussion leads to the following conclusion. In patterns like the ones in Figure 1 , the variables by which the visual system produces the impression of transparency are (1) the difference in reduction color on the two sides of the contour separating the filter from the background, $|\mathrm{p}-\mathrm{a}|$, and (2) the difference in color on the two sides of the contour delimiting the object inside the filter, $|p-q|$. Objects or parts of objects jutting out under the filter do not functionally enter the perceptual transparency mechanism (they enter indirectly by producing concomitant color alterations through simultaneous contrast and similar processes).

Let us now build a descriptive model to fit these results. Beck, Prazdny, and Ivry (1983) hypothesized 
that the computations carried out by the visual system in perceiving transparency were a linear function of lightness. On the basis of this hypothesis, it is assumed that apparent density varies linearly with the difference in reduction color. Let us assume that both apparent density and reduction color vary in the range of $[0,1]$. (Recall that observers spontaneously used a fixed range of numerical responses to rate apparent density). Let, therefore, the magnitude of apparent density of the filter evoked by the difference $|\mathrm{p}-\mathrm{a}|$ be $\psi_{\mathrm{p}, \mathrm{a}}=|\mathrm{p}-\mathrm{a}|$. In fact, the results in Figure 2a show that the lower the transmittance, and consequently the greater the difference $|p-a|$, the higher the apparent density. Let the magnitude of apparent density evoked by the difference $|p-q|$ be $\psi_{\mathrm{p}, \mathrm{q}}=1-|\mathrm{p}-\mathrm{q}|$. In fact, the results in Figure $2 \mathrm{a}$ show that apparent density increases as the reflectance of the figure approaches the reflectance of the background and, consequently, as the difference $|p-q|$ decreases.

The descriptive model proposed here states that the magnitude, $\psi$, of the overall impression of density of the filter is a weighted average of $\psi_{\mathrm{p}, \mathrm{a}}$ and $\psi_{\mathrm{p}, \mathrm{q}}$. In symbols,

$$
\psi=w|p-a|+(1-w)(1-|p-q|)
$$

where $w$ is an individual weight coefficient. For the three observers in Figure $2 b, w=1$. For the $19 \mathrm{ob}-$ servers in Figure 3, w fell close to 0 . For the 11 observers in Figure 3, w fell somewhere in between 0 and 1.

\section{EXPERIMENT 2}

This experiment had the purpose of looking for a simplification of Model 2. One important question was whether appropriate instructions were capable of rendering the individual weight coefficient $w$ equal to 0 or 1 . If so, one term in Model 2 would vanish.

\section{Method}

Observers. The observers were 10 students and members of the Institute of Psychology. They were not the same observers used in Experiment 1.

Stimuli. The stimulus conditions were the same as in Experiment 1 , except for the following changes. Three filters with a transmittance of $.1, .3$, or .5 were used. Sixteen backgrounds were built with a square in the middle. There were four sets of four backgrounds with the same reflectance. In each set, the reflectance of the square was varied, as indicated in Table 1.

Each background was always placed in the same position, so that the square on it was perceived in the middle of the filter (threesurface transparency).

Procedure. The procedure was the same as in Experiment 1, except for the following changes. Five observers were shown once each of the $\mathbf{4 8}$ combinations of the filter and the square (figure) on the background, and were asked to rate the apparent density of the filter over the figure. Then the $\mathbf{4 8}$ combinations were again shown once, and the observer was asked to rate the apparent density of the filter over the background while disregarding the figure. For the other five observers, this order was reversed. The rating
Table 1

Reflectances of the Squares Used in Experiment 2

\begin{tabular}{ccccc}
\hline $\begin{array}{l}\text { Reflectance of } \\
\text { Background }\end{array}$ & \multicolumn{4}{c}{ Reflectance of Squares } \\
\hline .04 & .06 & .13 & .35 & .76 \\
.19 & .23 & .31 & .52 & .87 \\
.35 & .31 & .26 & .19 & .08 \\
.76 & .67 & .52 & .31 & .13 \\
\hline
\end{tabular}

had to be performed using numbers in the range of $0-100$. The number 0 represented perfect transparency, as for example in the case of a colorless windowpane. The number 100 represented opacity. Before the rating began, the observer was given no information as to the range of apparent densities. As a single example of an experimental pattern, all observers were shown the same combination of filter (transmittance .3), square (reflectance .52), and background (reflectance .76).

\section{Results and Discussion}

The results are depicted in Figure 4. The upper four graphs refer to the estimate of apparent density over the background (unbroken lines). The lower graphs refer to the estimate of apparent density over the figure (dashed lines). No statistical test was applied since inspection of individual results showed that all observers produced substantially the same pattern of results as that in Figure 4.

The curves in the first upper graph superimpose around a density of 50 . These curves represent the means for nine observers. One observer was excluded from the computations, in this particular case, since all his numerical responses were practically 0 . Another observer declared that she could assign to a single filter 0 or 100 just as well. In point of fact, when a black filter is superimposed on a black background, a very ambiguous impression ensues. This, therefore, is a special case that should not be taken into account here.

As may be seen in Figure 4, the curves in the upper graphs are flatter than the curves in the lower graphs. However, the upper graphs are not as flat as the curves for the three observers in Figure $2 b$, whose $w=1$. Therefore, it may be concluded that the present instructions do have an effect on w. However, it seems that $w$ cannot be made exactly equal to 1 , and consequently, Model 2 cannot be simplified in the way hoped for.

\section{EXPERIMENT 3}

The purpose of this experiment was to check the assumption, on which Model 2 is based, that apparent density varies linearly with $|\mathrm{p}-\mathrm{a}|$ and $|\mathrm{p}-\mathrm{q}|$. This implies (Beck's hypothesis) that $\psi$ must vary linearly with $q$ if $p$ and $a$ are kept constant.

\section{Method}

Observers. The observers were 20 students and members of the Institute of Psychology, different from those used in Experiments 1 and 2. 

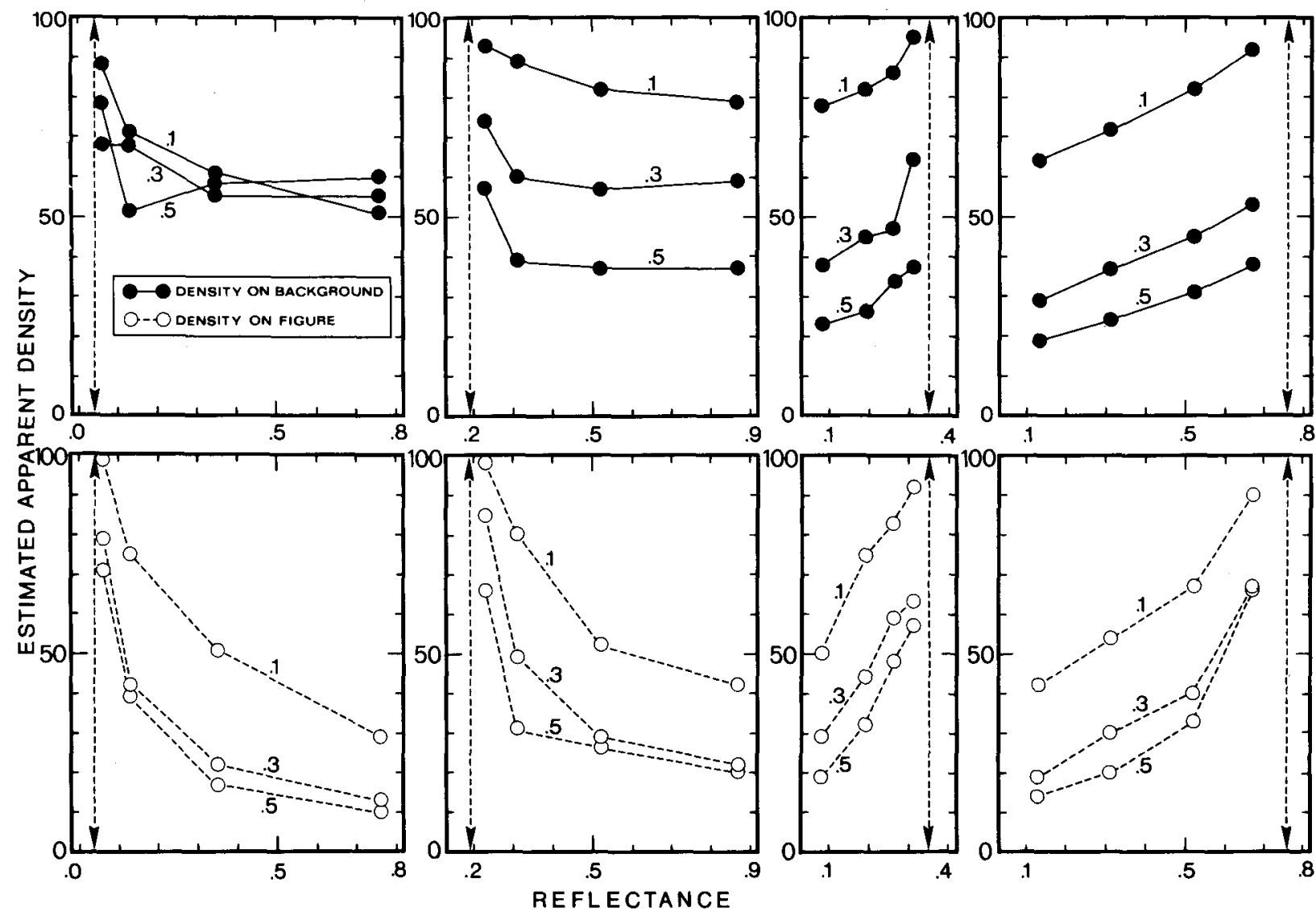

Figure 4. Estimated apparent density as a function of reflectance of a square on the background, for a transmittance of .1, .3, or .5. The reflectance of the background is indicated by the arrows. The upper (lower) graphs represent the results when the observer estimated the density on the background (figure).

Stimuli. This experiment was carried out in a different room. The stimulus conditions were the same as before, except for the following changes. The distance of the eye from the filter was $1.3 \mathrm{~m}$. The illumination level was $15 \mathrm{~lx}$. Three filters with a transmittance of $.1, .3$, or .8 were used. Eight backgrounds, all with the same reflectance, .23, were built. A square (figure) was stuck in the middle of each background. The reflectance of a square was $.26, .31, .35, .40, .46, .59, .67$, or .87 . Filters and figures were combined to produce 24 cases of three-surface transparency.

Procedure. The procedure was the same as before, except for the following changes. The observers were first shown once the 24 combinations of filter and figure, and asked to rate apparent density. Then they were again shown once the 24 combinations in a different random order, and asked to rate the whiteness of the figure as seen through the filter. Masin (in press) had found that, under these instructions, observers spontaneously estimated the reduction whiteness. The other 10 observers were first asked to rate whiteness, and then apparent density.

The observers had to use the numbers from 0 to 100 . In the case of apparent density, 0 represented perfect transparency and 100 , opacity. Numbers in between 0 and 100 had to be assigned to each filter proportionally. That is, the greater the density, the larger the number. In the case of whiteness, 0 represented the blackest black, and 100 the whitest white, ever experienced in the observer's life. Numbers in between had to be assigned to the whiteness of figures proportionally-that is, numbers lower than 50 to dark grays, numbers larger than 50 to light grays, and the number 50 to the figure having a gray color exactly between black and white. As a single example of a stimulus pattern, all observers were shown the same combination of filter (transmittance .3), and square (re- flectance .46) on the background. The observer was given no information as to the stimulus ranges. Each session lasted about $30 \mathrm{~min}$.

\section{Results and Discussion}

The results for each filter are depicted in Figure 5. The ratings of color density are represented on the ordinate, and the ratings of whiteness are represented on the abscissa. Using the least squares method, a straight line was fitted through the data points. The size of standard errors, represented by the vertical and horizontal bars, indicates that a straight line is, at least, a very good approximation of the truly fitting curve. This corroborates Beck's hypothesis.

Note that the slope of the straight line for the transmittance of .8 is consistently different from the slope for the transmittance of $.1[\mathrm{t}(19)=3.65, \mathrm{p}<.002]$. This shows that Model 2 is not additive (Anderson, 1970).

\section{CONCLUSION}

The results obtained in Experiment 1 (Figure 2a) show that the rated density of the transparent surface is functionally independent of the topological differ- 


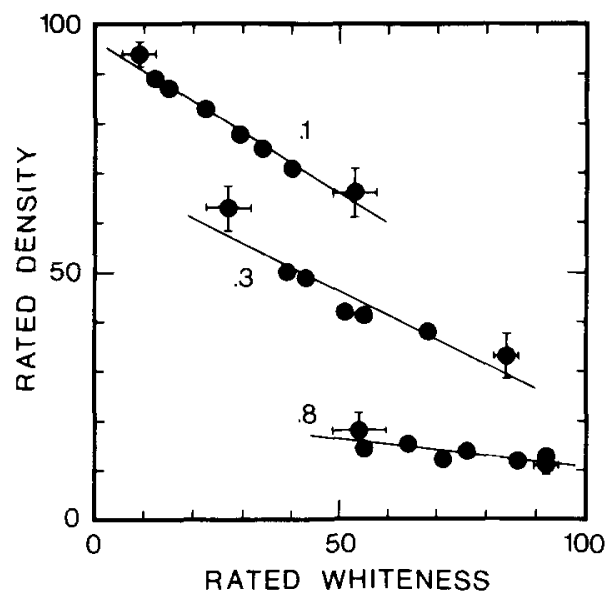

Figure 5. Relationship between apparent density of filter and whiteness of figure when, for a given filter with transmittance of $.1, .3$, or .8 , the difference in color between filter and background is kept constant.

ence between the three- and four-surface transparency. If Model 1 were developed to predict the rated density, these results would imply that Model 1 was not applicable even to the four-surface transparency. However, consider the important concept (Beck et al., 1983) that judgments of transparency may be based on stimulus relations that differ from those which determine whether the perception of transparency occurs. Metelli (1970, 1974b, 1975; Metelli, Masin, \& Manganelli, 1980) obtained experimental evidence proving that Model 1 was tenable at the ordinal level of measurement. This means that Model 1 actually encodes the all-or-none color relations (conditions) for the perception of transparency. Model 2 is an alternative to Model 1 insofar as it describes judgments of transparency. Model 2 does not necessarily encode the conditions that allow for the perception of transparency.

The results of Experiment 2 show that the weight, $w$, may be varied by means of proper instructions. However, the obtained variation is limited, showing reasonably that the individual differences are both perceptual and judgmental. In fact, it has been empirically found that Model 2 is not additive. Since $w$ varies with both $|p-a|$ and $|p-q|$ and with the attitude of the observer, predictions from Model 2 may be obtained only if the parameters of the individual functions relating $w$ to $|p-a|$ and $|p-q|$ are known in advance. This constitutes a weakness in Model 2.

This model, however, encodes relevant information as to the judgmental strategies employed by the observer in rating apparent density. When the observer evaluates apparent density, he seems to behave as an averaging mechanism. Given the inputs extracted from particular contours (the difference in reduction color between filter and figure and between filter and background), the observer gives an overall evaluation of apparent density by averaging the amount of apparent density that would be evoked separately by the input coming from each particular contour.

\section{REFERENCES}

Anderson, N. H. (1970). Functional measurement and psychological judgment. Psychological Review, 77, 153-170.

Beck, J., Prazdny, K., \& Ivry, R. (1983). The perception of transparency with achromatic colors. Unpublished manuscript, University of Oregon, Department of Psychology, Eugene.

Fuchs, W. (1923). Experimentelle Untersuchungen über das simultane Hintereinandersehen auf derselben Sehrichtung. Zeitschrift für Psychologie, 91, 145-235.

Gibson, J. J. (1975). Three kinds of distance that can be seen, or how Bishop Berkeley went wrong. In G. B. F. D'Arcais (Ed.), Studies in perception (pp. 83-87). Milan: Martello.

GyulaI, E. (1976). Grado, colore e uniformità della trasparenza fenomenica. Atti dell'Istituto Veneto di Scienze, Lettere ed Arti, 134, 791-803.

KanizsA, G. (1979). Organization in vision. New York: Praeger. Katz, D. (1911). Erscheinungsweisen der Farben und ihre Beeinflussung durch die individuelle Erfahrung. Leipzig: Barth.

LAUENSTEIN, O. (1943). Schattencharakter und Dingcharakter. Zeitschrift für Psychologie, 154, 203-214.

Masin, S. C. (1978). A contribution to the theory of perceptual scission in phenomenal transparency. Italian Journal of Psychology, 5, 171-191.

Masin, S. C. (in press). A psychophysical study of the Fuchs phenomenon. Perception, in press.

Masin, S. C., \& Idone, A. M. (1980). Studio sperimentale sulla percezione della trasparenza con figura e sfondo acromatici e omogenei. Giornale Italiano di Psicologia, 8, 265-277.

Metelli, F. (1970). An algebraic development of the theory of perceptual transparency. Ergonomics, 13, 59-66.

MEtelLi, F. (1974a). The perception of transparency. Scientific American, 230(4), 90-98.

Metelli, F. (1974b). Achromatic color conditions in the perception of transparency. In R. B. Macleod \& H. L. Pick (Eds.), Perception (pp. 95-116). Ithaca, NY: Cornell University Press.

MetelLI, F. (1975). On the visual perception of transparency. In G. B. F. D'Arcais (Ed.), Studies in perception (pp. 445-487). Milan: Martello.

METELLI, F. (1982). Stimulation and perception of transparency (Report No. 73). Padua, Italy: University of Padua, Institute of Psychology.

Metelli, F., Masin, S. C., \& Manganelli, A. M. (1980). Partial transparency. Atti e Memorie dell'Accademia Patavina di Scienze, Lettere ed Arti, 92, 115-169.

Moore-Heider, G. (1933). New studies in transparency, form, and colour. Psychologische Forschung, 17, 13-55.

Tudor-HART, B. (1928). Studies in transparency, form, and color. Psychologische Forschung, 10, 255-298.

\section{NOTES}

1. The reduction color corresponding to a given region of a transparent pattern (i.e., $Q$ in Figures $1 \mathrm{a}$ or $1 \mathrm{~b}$ ) is the color (1) when the region is looked at while assuming an extremely analytical attitude (Fuchs, 1923), (2) when transparency is abolished by altering the so-called topological or figural conditions of transparency (Kanizsa, 1979), or (3) when the region is looked at through the hole of a reduction screen producing, in the hole, the same amount of simultaneous contrast as that produced, in the region looked at, when there is no reduction screen.

2. In point of fact, Metelli developed the model using reflectances in place of reduction colors, thus producing a physical 
model of transparency. Recently, however, he (Metelli, 1982) has used only reduction colors.

3. In the special case in which the degree of transparency and the color of the transparent surface are phenomenally homogeneous over the entire surface, $\alpha=\alpha_{1}=\alpha_{2}$ and $t=t_{1}=t_{2}$, and the system of equations constituting Model 1 can be solved for both $\alpha$ and $\mathrm{t}$ (Metelli, 1982).

4. Katz (1911) proposed a similar explanation for the perception of mist. According to Katz, when one looks through a filter covering the entire visual field, an impression of mist ensues. The thickness of the mist increases as the transmittance of the filter decreases. The condition for the diminution of the thickness of the mist would be the reduction in clearness, or sharpness, of the surface contours.

The results of an experiment by Gyulai (1976) also stress the importance of the difference in reflectance between adjacent proximal surfaces. In her experiment, patterns made of gray paper were used. The results can be rephrased as follows. If episcotisters with the same sector size have colors varying from black to white, and the same bicolored background is looked at through each episcotister revolving at fusion speed, the observers, who are asked to arrange the episcotisters in order of increasing apparent density, produce two opposite dispositions. Half the observers order the episcotisters from black to white, and half the observers do so from white to black. The occurrence of a given disposition depends upon which part of the background the observer chooses to look at through the episcotister. The less the difference in (reduction) color between the background and the episcotister, the less the judged density. Gyulai did not extent the experiment to episcotisters with different sector sizes.

(Manuscript received October 31, 1983; revision accepted for publication February 23, 1984.) 